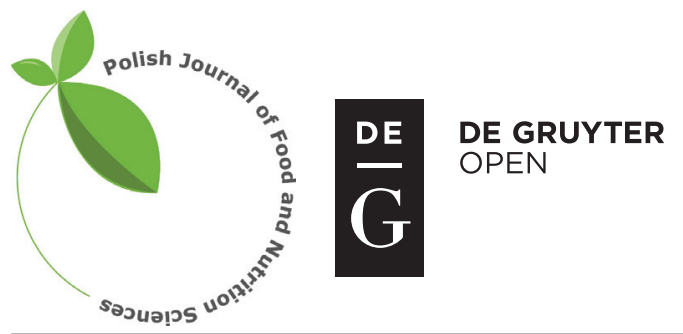

Pol. J. Food Nutr. Sci., 2018, Vol. 68, No. 1, pp. 15-23

DOI: $10.1515 /$ pjfns-2017-0013 http://journal.pan.olsztyn.pl

Original research article

Section: Food Technology

\title{
Optimization of Processing Parameters for Lettuce Vacuum Osmotic Dehydration Using Response Surface Methodology
}

\author{
Yuejin Yuan ${ }^{1 *}$, Libin Tan ${ }^{1}$, Yingying Xu ${ }^{1}$, Jixian Dong ${ }^{1}$, Yu Zhao ${ }^{1}$, Yueding Yuan ${ }^{2}$ \\ ${ }^{1}$ College of Mechanical and Electrical Engineering, Shaanxi University of Science and Technology, 6 Xuefuzhong Road, \\ Weiyangdaxueyuan district of Xi'an, 710021, China \\ ${ }^{2}$ College of Mathematics and Computer Science, Yichun University, 336000, China
}

Key words: lettuce, vacuum osmotic dehydration, regression mathematical model, response surface methodology, processing parameters optimization

\begin{abstract}
In order to obtain the optimal technological parameters of lettuce vacuum osmotic dehydration, the effects of osmotic temperature, slice thickness, sucrose concentration, and vacuum degree on the vacuum osmotic dehydration were explored. The lettuce water loss rate and solid gain rate decreased with the increase of slice thickness and vacuum degree, and increased with the increase of sucrose concentration and osmotic temperature. Response surface methodology was applied to analyze the influence of the four influential factors on the evaluated parameters and the optimization of lettuce vacuum osmotic dehydration was studied. The results indicated that, within the experimental scope, the optimized technological parameters of lettuce vacuum osmotic dehydration are the temperature of $28^{\circ} \mathrm{C}$, the slice thickness of $2 \mathrm{~mm}$, sucrose concentration of $47 \%$, the vacuum degree of $22 \mathrm{kPa}$, and the water loss rate and solid gain rate are $72.16 \%$ and $11.82 \%$, respectively.
\end{abstract}

\section{INTRODUCTION}

Lettuce (Lactuca sativa) is one of the most consumed vegetables in many parts of the world, being known for its high nutritional value and delicious taste, and high contents of vitamins and minerals. Therefore, lettuce is widely beloved by the majority of people [Zhang et al., 2012]. Prior to the beginning of the drying processes of lettuce, osmotic dehydration is often used as a pre-treatment method for removing most of water from solid food. In this way, the quality of dried products can be improved and meanwhile, the total energy consumption for drying and the drying time are reduced [Dong \& Chen, 2004]. The main principle of osmotic dehydration is described as follows: at first, the experimental materials (fruits or vegetables) should be put in the high osmotic pressure solution with a certain temperature. Due to the characteristics of semi-permeability of the cell membrane of the material, and the osmotic pressure difference at both ends of the cell membrane, the water inside the cell membrane enters into the osmotic solution through the cell membrane, so as to achieve the purpose of removing the partial water of the material [Souraki et al., 2012; Abraão et al., 2013; Chandra \& Kumari, 2015; Goula \& Lazarides, 2012].

Generally, the rate of water removal through the method of osmotic dehydration is very slow. Therefore, many auxil-

\footnotetext{
* Corresponding Author: Tel.: +86-029-86195629; Fax: +86-02986168302;

E-mail: yyjyuan1@163.com
}

iary methods were used to accelerate the dehydration process without changing the original characteristics of the materials [Xin et al., 2013; Nair et al., 2015; Nieto et al., 2012; Nowacka et al., 2014]. Vacuum osmotic dehydration has been widely concerned and vigorously promoted by researchers because of its high dehydration rate, high nutrient retention rate, and small environmental pollution [Zhao et al., 2014]. At present, there are many research studies on the processing of fruits and vegetables by applying the method of vacuum osmotic dehydration, such as carrot, mango, peach, apricot [Wray \& Ramaswamy, 2015; Corrêa et al., 2014; Yadav et al., 2012; Azam et al., 2013; Huang \& Fan, 2013]. However, the studies on vacuum osmotic dehydration of lettuce are sparse in the open literature.

In recent years, response surface methodology (RSM) has been extensively applied for optimization study of test factors and obtained response surfaces [Acosta et al., 2008; Ghosh \& Das, 2014; Lazo-Vélez et al., 2016]. This method has been successfully applied by several researchers to determine the optimum parameters [Bejaouiet al., 2016; Yang et al., 2016; Kaushik et al., 2016; Erbay et al., 2015; Saxena et al., 2015; Esan et al., 2015]. The major advantages of RSM is that it offers a large amount of information from a relative small number of experiments, allowing the observation of both the effect of the independent variables on the response as well as their possible interactions. Therefore, this study intended to take the lettuce as the research object, to investigate the effects of the main influencing factors on the vacuum osmotic dehydration processes and to obtain the optimum technological parameters of lettuce 
vacuum osmotic dehydration by applying the regression analysis and response surface methodology. The results of this study can provide the technical basis for the production of lettuce and other related fruits and vegetables using vacuum osmotic dehydration, and also provide the design basis for the research and development of the vacuum osmotic dehydration equipment for the fruits and vegetables.

\section{MATERIALS AND METHOD}

\section{Materials and equipment}

The fresh lettuce, purchased from a local market in Xi'an, was used as the raw material in the experiments. For each experiment, the lettuce was peeled, washed in running tap water, and cut into slices. The thickness of slices was $2 \mathrm{~mm}, 4 \mathrm{~mm}$, and $6 \mathrm{~mm}$. The reagents used in this study included: sucrose (the osmotic agent, a food grade commercial granulated cane sugar purchased from a local supermarket in Xi'An China), sodium chloride (food grade, Tianjin Zhiyuan Chemical Reagent Co., Ltd, Tianjin, China), ascorbic acid (food grade, Zhengzhou Tuoyang Industrial Co., Ltd, Henan, China), citric acid (food grade, Shandong Weifang Yingxuan Industry Co., Ltd, Shandong, China), and anhydrous calcium chloride. (food grade, Henan Dongcheng Food Chemical Co., Ltd, Henan, China).

The experimental apparatuses used in the experiment included: an electric thermostatic drying oven (DHG 9240A type, Shanghai Pudong Rongfeng Scientific Instrument Co., Ltd, Shanghai, China), electronic balance (Shanghai Youke Instrument Co., Ltd, Shanghai, China, accuracy to $0.001 \mathrm{~g}$ ), and self-made vacuum equipment (as shown in Figure 1).

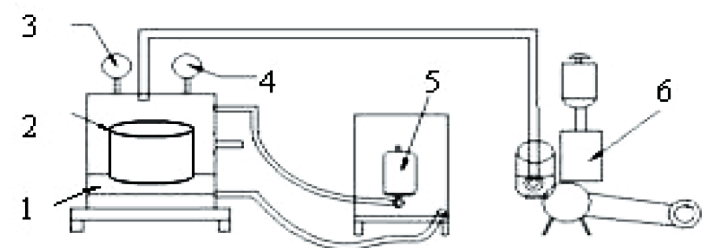

FIGURE 1. Sketch of lettuce vacuum osmotic dehydration test equipment. 1. vacuum chamber; 2. osmotic dehydration tank; 3. vacuum gauge; 4. thermometer; 5 . heating system; 6 . vacuum pumping system.

\section{Evaluation index}

During osmotic dehydration process, the water loss and solid gain take place simultaneously. The mass of the lettuce slices was reduced due to water loss, but at the same time, there was an increase in the mass due to solid gain. The mass exchange between the solution and samples was evaluated by using the parameters such as water loss rate (WL) and solid gain rate (SG). These two parameters were chosen as the experimental evaluation parameters, and calculated by the following equations:

$$
S G=\frac{W_{s t}-W_{s o}}{W_{o}} \times 100 \%
$$

$$
W L=\frac{W_{m o}-W_{m t}}{W_{o}} \times 100 \%
$$

where: Wst is the final solid mass of material (g); Wso is the initial solid mass of material (g); Wo is the initial total mass of material $(\mathrm{g})$; Wmo is the initial mass of water content in material $(\mathrm{g})$; and $W m t$ is the final mass of water content in material (g). Generally, the greater water loss rate is good, while the smaller increase in solid gain rate is better. This is mainly because the greater the solid gain rate is, the more solute sucrose entered into the lettuce samples, which makes that the lettuce will become sweet and that its original flavor will change as well.

\section{Experimental procedures}

For each experiment of osmotic dehydration, the main experimental steps were as follows. At first, the fresh lettuce used as the research object in this study was peeled and washed, and then cut into slices. The thickness of slices was $2 \mathrm{~mm}$, $4 \mathrm{~mm}$, and $6 \mathrm{~mm}$. Then, the initial parameters of lettuce (Wmo, Wso, Wo ) were measured before the beginning of osmotic dehydration experiment. With the completion of preparatory work for the experiment, the osmotic dehydration experiments were classified into groups. The desired concentration of osmotic solution for the experiment of osmotic dehydration was prepared and the known mass of lettuce samples were immersed in the osmotic solutions, indicating that the osmotic dehydration experiment began. During the osmotic treatment, every half-an-hour the lettuce samples were removed from osmotic solution and weighed after removing the solution adhering to the surface using the filter paper, and this procedure was continued until the mass of the samples remained constant. At the end of the osmotic dehydration processes, the final parameters of lettuce samples (Wst, Wmt) were obtained. All the experiments were performed in triplicate, and the average value was used for the determination of water loss rate and solid gain rate.

During the experiment, the lettuce samples were firstly immersed in an osmotic dehydration tank with a solid to liquid ratio of 1:10. Then the heating procedure was started to maintain the osmotic solution temperature in the osmotic dehydration tank, followed by controlling the vacuum pumping procedure start with an interval time, to make sure that the vacuum state (5 min) and ambient pressure state $(25 \mathrm{~min}$ ) appeared alternatively in the vacuum chamber.

\section{Single factor experiments}

In order to investigate the effect of a specific parameter on the experimental results, the single factor experiments were conducted by applying the control variables method, which means that there is only one variable among the influencing factors on the experimental results, and the other variables are kept constant [Dak \& Pareek, 2014]. The influence of the osmotic temperature, slices thickness, sucrose concentration and vacuum degree on lettuce vacuum osmotic dehydration processes was studied in our work. Each time, $100 \mathrm{~g}$ of the prepared lettuce samples have been processed for a single factor experiment. The process parameters and their values which are not specified are listed out as follows: os- 
TABLE 1. Levels and codes of experimental factors.

\begin{tabular}{l|ccccc}
\hline Code & $\begin{array}{c}\text { Osmotic } \\
\text { temperature } \\
\left(\mathrm{X}_{1} /{ }^{\circ} \mathrm{C}\right)\end{array}$ & $\begin{array}{c}\text { Vacuum } \\
\text { degree } \\
\left(\mathrm{X}_{2} / \mathrm{kPa}\right)\end{array}$ & $\begin{array}{c}\text { Slice } \\
\text { thickness } \\
\left(\mathrm{X}_{3} / \mathrm{mm}\right)\end{array}$ & $\begin{array}{c}\text { Sucrose } \\
\text { concentration } \\
\left(\mathrm{X}_{4} / \%\right)\end{array}$ \\
\hline$+\gamma$ & 40 & 20 & 2 & $50 \%$ \\
+1 & 36 & 24 & 3 & $46 \%$ \\
0 & 30 & 30 & 4 & $40 \%$ \\
-1 & 24 & 36 & 5 & $34 \%$ \\
$-\gamma$ & 20 & 40 & 6 & $30 \%$ \\
\hline
\end{tabular}

motic temperature $20^{\circ} \mathrm{C}$, slice thickness $2 \mathrm{~mm}$, the mass fraction of sucrose solution $40 \%$, and vacuum degree $20 \mathrm{kPa}$.

\section{Quadratic regression orthogonal rotation combination experiments}

Quantitative experimental data can be obtained from properly designed experiments. Currently, the orthogonal combination experiments are identified as the most effective method for analyzing the interaction effect of different influential factors [Maran et al., 2013]. Therefore, the $\mathrm{L}_{23}\left(3^{4}\right)$ orthogonal table was used in the orthogonal combination test and a total of 23 runs were conducted in our research work. Each time, $100 \mathrm{~g}$ of the prepared lettuce samples have been used for one orthogonal combination test. The orthogonal combination experiments were conducted by considering the osmotic temperature, sucrose concentration, vacuum degree, and slices thickness as the four independent variables, and the water loss and solid gain as the two evaluation parameters. The levels and codes of experimental factors were shown in Table 1.

\section{Statistical analysis}

In this work, in order to ensure the accuracy, reliability and repeatability of the obtained experimental data, all the tests for the measurement of experimental data were replicated three times. The average values of the measurements of these replications were used for statistical analysis to determine the effects of osmotic temperature, slices thickness, sucrose concentration, and vacuum degree (four influence factors) on the water loss rate and solid gain rate (two experimental evaluation parameters). Standard deviations for statistical analysis were estimated by means of Microsoft Excel 2003 (Microsoft Corporation, Redmond, WA). Furthermore, in order to obtain the optimized technological parameters of vacuum osmotic dehydration of lettuce, the regression analysis was conducted by statistical analysis with the help of SPSS 17.0 software (IBM Corporation, Armonk, NY). The results of statistical analysis are described subsequently in details.

\section{RESULTS AND DISCUSSION}

\section{Single factor experiments}

\section{The influence of osmotic temperature}

The effect of different osmotic temperatures on the rate of water loss and solid gain of lettuce samples was shown in Figure 2. It can be seen from the figure that the higher the temperature, the higher the diffusion rate for the sucrose to enter into the lettuce slices. While, in the same operating conditions, the effect of temperature on the water loss rate was not so significant. The water loss rate under the osmotic temperature of $30^{\circ} \mathrm{C}$ was slightly higher than the osmotic temperature $20^{\circ} \mathrm{C}$, and the water loss rate under the osmotic temperature $40^{\circ} \mathrm{C}$ was also only slightly higher than the water loss rate of osmotic temperature $30^{\circ} \mathrm{C}$.

\section{The influence of slice thickness}

The impact of slice thickness on the rate of water loss and solid gain was shown in the Figure 3. Both water loss rate and solid gain rate increased with the decrease of slice thickness. The thinner the slice thickness, the grater the rate of water loss and solid gain. The increasing tendency for both two experimental evaluation parameters was almost similar. At the early stage of osmotic dehydration process, the thinnest lettuce slice created the greatest rate of water loss, and the difference compared with others was quite significant. It can be concluded that the smaller the slice thickness of lettuce can save the time of osmotic dehydration. To the end stage of osmotic dehydration process of lettuce, the rate of water loss under different slice thickness was close. In conclusion, the smaller slice thickness of material creates the better dehydration if the operating condition is allowed.

\section{The influence of sucrose concentration}

The effect of sucrose concentration on the rate of water loss and solid gain of lettuce was shown in Figure 4. Sucrose concentration is one of the key factors that influence the experimental results during the osmotic dehydration process of lettuce. Generally, the dehydration efficiency can be obtained as high as possible under the premise of ensuring the permeation of solutes into the material as little as possible. As shown in Figure 4, it is concluded that the higher the sucrose concentration of the osmotic solution, the greater the rate of water loss of lettuce. Meanwhile, compared with the water loss rate of lettuce under the condition that the osmotic solution which sucrose concentration is $30 \%$, the water loss rate of lettuce under the condition that the osmotic solution which sucrose concentration is $40 \%$ has a greater increment, but the difference between the water loss rate of lettuce under the condition that osmotic solution which sucrose concentration is $40 \%$ and $50 \%$ respectively is quite small. Accordingly, the solid gain rate increases with the increase of sucrose concentration. Therefore, in order to obtain high-quality dehydrated products, the bigger water loss rate and smaller solid gain rate should be obtained through adopting the optimized sucrose concentration parameter of osmotic solution for dehydration process, while not just blindly increase sucrose concentration of osmotic solution for the reason that higher sucrose concentration cause higher solid gain rate.

\section{The influence of vacuum degree}

The effect of vacuum degree on the water loss rate and the increase rate of the solid is shown in Figure 5. It was found that the higher the vacuum degree, the smaller the rate of water loss. The osmotic dehydration efficiency of let- 

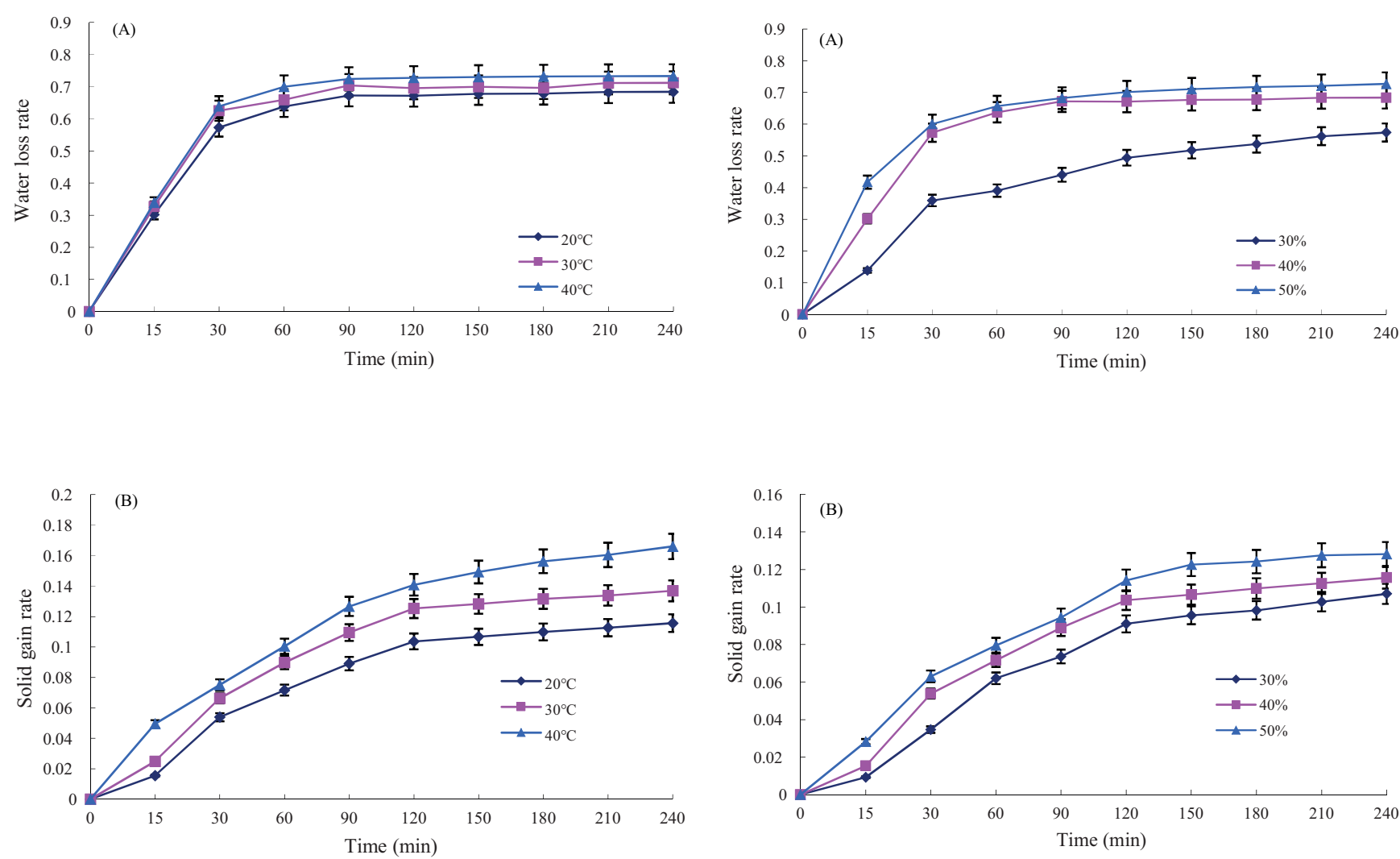

FIGURE 2. Effects of osmotic temperature on water loss rate (A) and solid gain rate $(\mathrm{B})$.

FIGURE 4. Effects of sucrose concentration on water loss rate (A) and solid gain rate $(\mathrm{B})$.
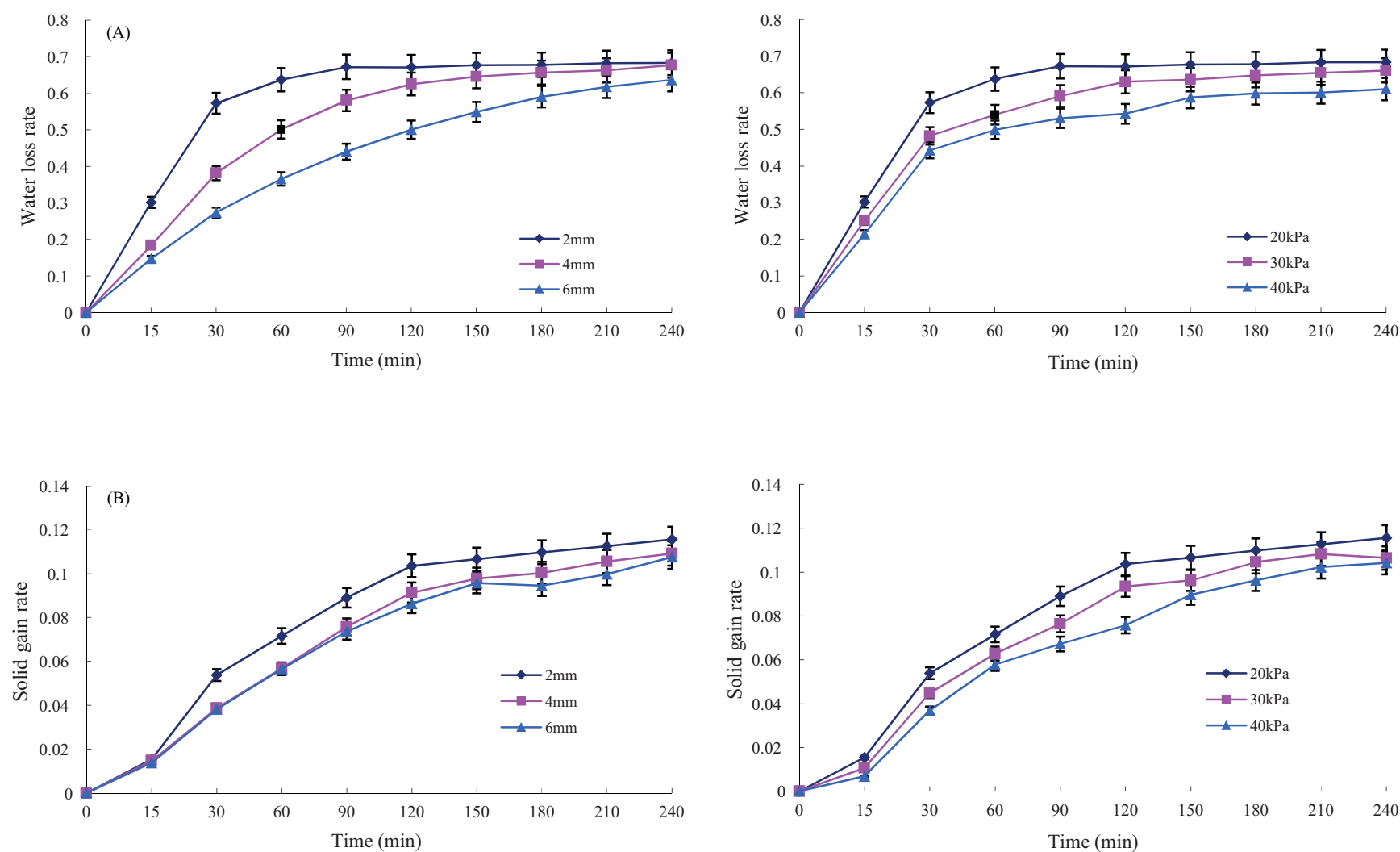

FIGURE 3. Effects of slice thickness on water loss rate (A) and solid gain rate (B).

FIGURE 5. Effects of vacuum degree on water loss rate (A) and solid gain rate (B). 
tuce under the vacuum degree of $20 \mathrm{kPa}$ was obviously improved when compared with the vacuum degree of $30 \mathrm{kPa}$ and $40 \mathrm{kPa}$. The rate of water loss was higher under the condition of the vacuum degree of $20 \mathrm{kPa}$. At the initial period of osmotic dehydration process, the rate of water loss increased rapidly. And then the increasing tendency was stable till the end of osmotic dehydration process. This phenomenon has also occurred under the vacuum degree of $30 \mathrm{kPa}$ and $40 \mathrm{kPa}$, which indicates that effect of the vacuum degree on water loss rate can be quickly revealed. As for the effect of vacuum degree on the increase of solid gain rate, it can be seen from the growth trend of the solid gain that the increase rate of solid gain was the greatest under the condition of the vacuum degree of $20 \mathrm{kPa}$.

\section{Quadratic regression orthogonal rotation combination experiments}

\section{Regression analysis}

In order to minimize the effects of unexplained variability in the observed responses due to extraneous factors, the orthogonal rotation combination experiments were randomized. According to the order of the randomized combination experiments, the experimental results on the four independent variables, namely the osmotic temperature, slices thickness, sucrose concentration and vacuum degree on the two evaluation parameters are shown in Table 2. The $\mathrm{X}_{1}, \mathrm{X}_{2}, \mathrm{X}_{3}$ and $\mathrm{X}_{4}$ were the encoded values of the experimental factors, namely osmotic temperature, vacuum degree, slice thickness, and sucrose concentration respectively.

The SPSS 17.0 software (IBM Corporation, Armonk, $\mathrm{NY}$ ) was used for the regression analysis and the obtained regression equations for water loss rate and solid gain rate are given below:

$$
\begin{aligned}
Y_{1}= & 71.483+0.203 X_{1}-0.476 X_{2}-1.141 X_{3}+1.346 X_{4} \\
& +0.136 X_{1}^{2}+0.352 X_{1} X_{4}-0.116 X_{2}^{2}-0.785 X_{3}^{2}
\end{aligned}
$$

$$
\begin{aligned}
Y_{2}= & 12.416+1.253 X_{1}-0.486 X_{2}-1.479 X_{3}+0.872 X_{4} \\
& -0.115 X_{2}^{2}+0.576 X_{1} X_{4}-0.102 X_{3}^{2}
\end{aligned}
$$

Through regression analysis and analysis of variance for regression equation $Y_{1}$ (water loss rate) and regression equation $\mathrm{Y}_{2}$ (solid gain rate), it was found that the water loss rate of lettuce was significantly affected by four processing parameters of vacuum osmotic dehydration for the reason that the $\mathrm{P}$ value of significance test for regression equation $\mathrm{Y}_{1}$ was 0.0008 , which is in the significance range of $0-0.01$. The $P$ value of lack of fit test for regression equation $\mathrm{Y}_{1}$ is 0.1819 , indicating that the lack of fit results was not significant and proving that the established regression model for water loss rate is proper. Similarly, the $\mathrm{P}$ value of significance test and lack of fit for the regression equation $\mathrm{Y}_{2}$ (solid gain rate) were 0.0004 and 0.1263 , respectively. It indicated that the ef-

\begin{tabular}{|c|c|c|c|c|c|c|}
\hline \multirow{2}{*}{ No. } & \multicolumn{4}{|c|}{ Experimental design } & \multicolumn{2}{|c|}{ Results } \\
\hline & $X_{1}$ & $X_{2}$ & $X_{3}$ & $\mathrm{X}_{4}$ & $\begin{array}{l}\text { Water loss } \\
\text { rate } \mathrm{Y}_{1} / \%\end{array}$ & $\begin{array}{l}\text { Solid gain } \\
\text { rate } \mathrm{Y}_{2} / \%\end{array}$ \\
\hline 1 & 1 & 1 & 1 & 1 & 71.12 & 14.22 \\
\hline 2 & 1 & 1 & -1 & -1 & 76.16 & 14.04 \\
\hline 3 & 1 & -1 & 1 & -1 & 65.47 & 12.56 \\
\hline 4 & 1 & -1 & -1 & 1 & 79.11 & 14.16 \\
\hline 5 & -1 & 1 & 1 & -1 & 64.11 & 12.16 \\
\hline 6 & -1 & 1 & -1 & 1 & 70.57 & 12.28 \\
\hline 7 & -1 & -1 & 1 & 1 & 68.43 & 11.54 \\
\hline 8 & -1 & -1 & -1 & -1 & 69.89 & 11.96 \\
\hline 9 & 1.682 & 0 & 0 & 0 & 70.68 & 13.98 \\
\hline 10 & -1.682 & 0 & 0 & 0 & 65.42 & 12.06 \\
\hline 11 & 0 & 1.682 & 0 & 0 & 68.77 & 12.24 \\
\hline 12 & 0 & -1.682 & 0 & 0 & 69.54 & 12.04 \\
\hline 13 & 0 & 0 & 1.682 & 0 & 66.79 & 12.2 \\
\hline 14 & 0 & 0 & -1.682 & 0 & 72.99 & 13.42 \\
\hline 15 & 0 & 0 & 0 & 1.682 & 73.46 & 13.02 \\
\hline 16 & 0 & 0 & 0 & -1.682 & 62.89 & 11.22 \\
\hline 17 & 0 & 0 & 0 & 0 & 69.72 & 11.61 \\
\hline 18 & 0 & 0 & 0 & 0 & 69.71 & 11.59 \\
\hline 19 & 0 & 0 & 0 & 0 & 69.69 & 11.62 \\
\hline 20 & 0 & 0 & 0 & 0 & 69.74 & 11.62 \\
\hline 21 & 0 & 0 & 0 & 0 & 69.77 & 11.61 \\
\hline 22 & 0 & 0 & 0 & 0 & 69.76 & 11.62 \\
\hline 23 & 0 & 0 & 0 & 0 & 69.77 & 11.62 \\
\hline
\end{tabular}
fect of processing parameters of vacuum osmotic dehydration on solid gain rate was highly significant and the estab-
TABLE 2. Design and results of orthogonal experiment.

lished regression model for solid gain rate was proper too. The correlation coefficients for water loss rate and solid gain rate were 0.912 and 0.904 , respectively, which indicated that the two quadratic regression models could explain the change of the response values of $91.2 \%$ and $90.4 \%$, respectively. According to the above analysis, we can conclude that the regression models enable analyzing and predicting the water loss rate and solid gain rate perfectly for the vacuum osmotic dehydration of lettuce.

\section{Response surface analysis}

Response surface plots as a function of two factors at a time, maintaining all other factors at fixed or zero level are more helpful in understanding both the main and interactive effects of these two factors. The response surface curves were plotted to understand the interaction of each variable for maximum response. The graphical presentations for water loss rate and solid gain rate developed as a simultaneous function of the two independent variables according to their 

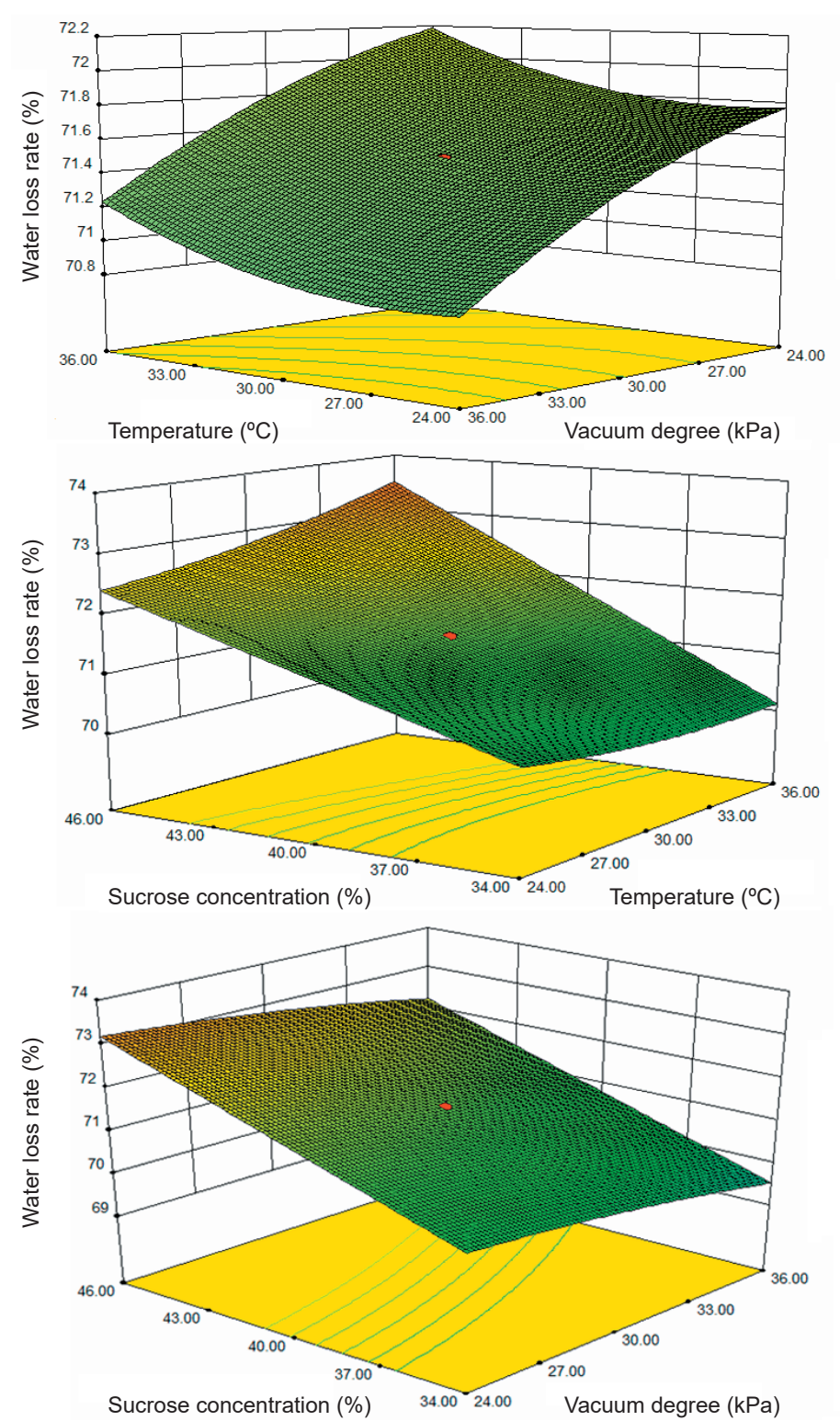

FIGURE 6. Response surfaces of the water loss rate.

significance to the response are provided in Figure 6 and Figure 7, respectively.

Figure 6 shows the response surfaces of water loss rate. Based on results depicted in the the figure, we can conclude that the strength order of the four influential factors on water loss rate is: sucrose concentration, slice thickness, vacuum degree, and temperature. Figure 7 shows the response surfaces of solid gain rate. The sucrose concentration affected the solid gain rate the most, while the vacuum degree the least. The strength order of the four independent variables on the solid gain rate is: sucrose concentration, temperature, slice thickness, and vacuum degree. Results of the response surface analysis obtained in this part are merely valid within the limits of the experimental factors used, any other extrapolation for responses beyond the range of the four dependent variables may be invalid.

\section{Optimization of processing parameters}

Optimization of the process parameters for lettuce vacuum osmotic dehydration was carried out to obtain the desired
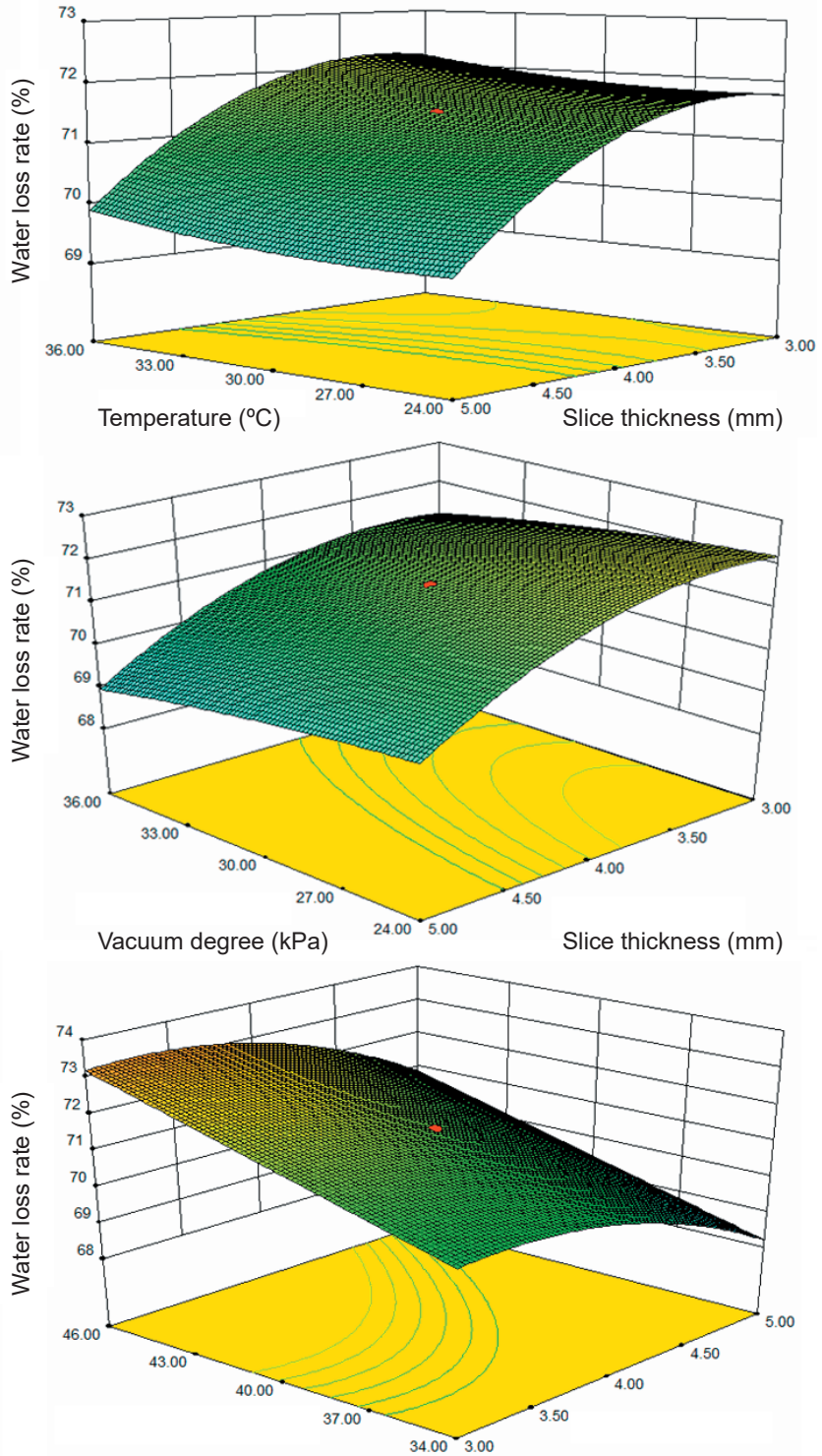

Sucrose concentration (\%)

Slice thickness $(\mathrm{mm})$

criteria for each response. Usually, several response variables describing the quality characteristics and all the processing parameters of processing procedure are to be optimized. The independent variables were kept in the experimental range while the responses were either maximized or minimized. Because of these responses are always competing in many cases, improving one response may have an opposite effect on another one, which further complicates the problem. Therefore, it is necessary to find the optimum conditions based on the desirable characteristics of the vacuum osmotic dehydration quality of lettuce.

As described in the earlier part of this paper, the osmotic dehydration process is considered as good when the water loss rate is high and the rate of solid gain is low during the vacuum osmotic dehydration of lettuce. But in the section of single factor experiments, it was found that the increase of osmotic temperature and sucrose concentration can increase the water loss rate and solid gain rate. Therefore, in order to obtain the optimal processing parameters for the lettuce vacuum 

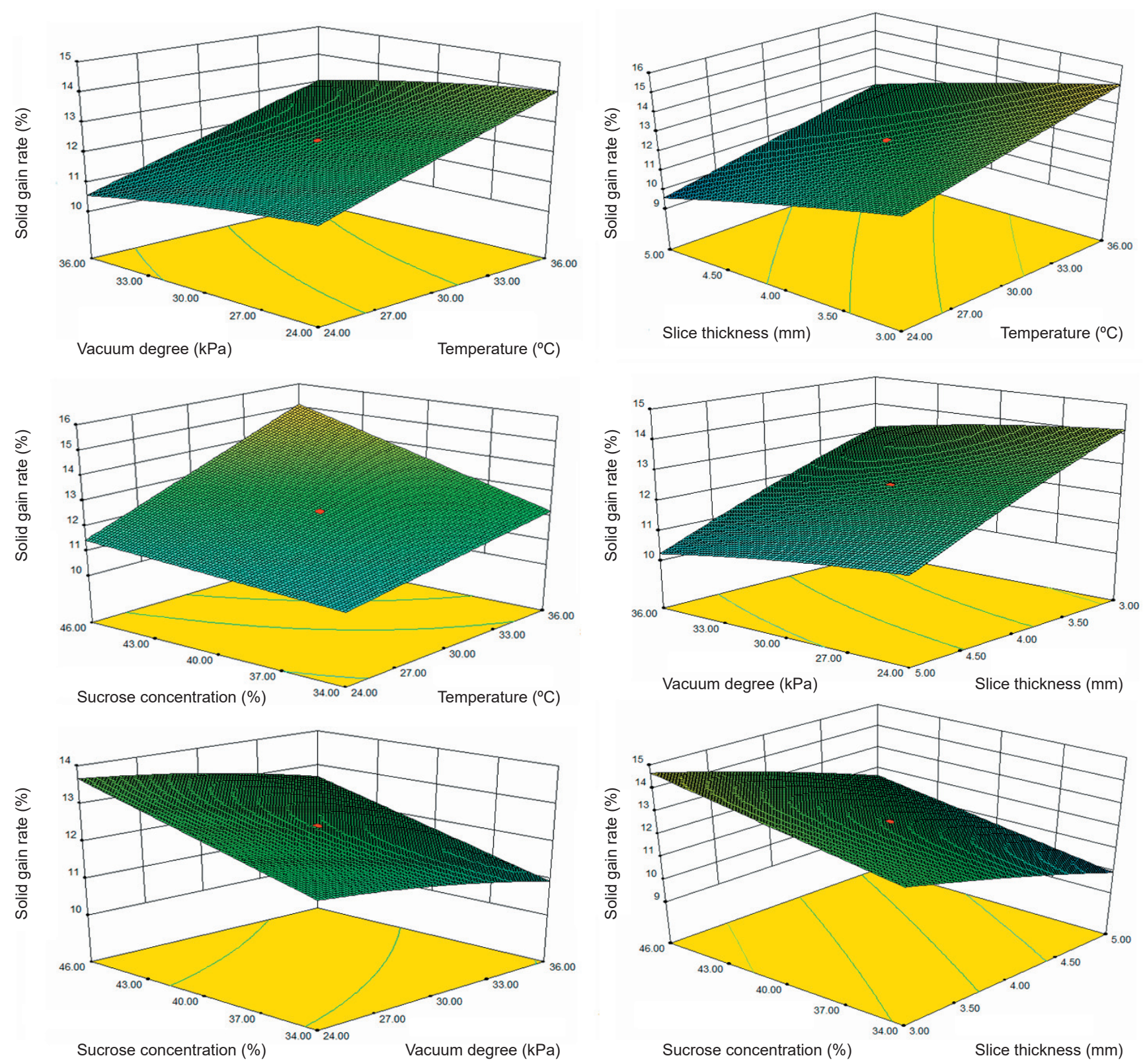

FIGURE 7. Response surfaces of the solid gain rate.

osmotic dehydration, the multi-objective non-linear optimization method was applied to the two objective functions and the best drying process parameters that satisfied the two functions also could be calculated.

The objective functions were:

$$
\left\{\begin{array}{l}
Y_{1} \rightarrow Y_{1 \max } \\
Y_{2} \rightarrow Y_{2 \min }
\end{array}\right.
$$

The constraint conditions were:

$$
\begin{cases}Y_{j} \geq 0 & (j=1,2) \\ -1.682 \leq X_{i} \leq 1.682 & (i=1,2,3,4)\end{cases}
$$

The optimization results obtained by the analysis software indicated that the osmotic temperature of $28^{\circ} \mathrm{C}$ (en- coded value -0.403), the slice thickness of $2 \mathrm{~mm}$ (encoding value -1.682), sucrose concentration of $47 \%$ (encoded value 1.273 ), and the vacuum degree of $22 \mathrm{kPa}$ (encoded value -1.409) were the optimum technological parameters for the vacuum osmotic dehydration of lettuce. At these optimal conditions, the water loss rate was $72.16 \%$, and solid gain rate was $11.82 \%$.

\section{CONCLUSION}

In this study, the effects of four processing parameters (osmotic temperature, slice thickness, sucrose concentration, and vacuum degree) on the lettuce vacuum osmotic dehydration were studied. Meanwhile, the optimal processing parameters were obtained through response surface methodology. The main results can be summarized as follows:

1. Both the water loss rate and solid gain rate decreased with the increase of slice thickness and vacuum degree, and in- 
creased with the increase of osmotic temperature and sucrose concentration.

2. The optimum technological parameters for the vacuum osmotic dehydration of lettuce were the temperature of $28^{\circ} \mathrm{C}$, the slice thickness of $2 \mathrm{~mm}$, sucrose concentration of $47 \%$ and the vacuum degree of $22 \mathrm{kPa}$. At these optimal conditions, the water loss rate was $72.16 \%$, and solid gain rate was $11.82 \%$.

\section{ACKNOWLEDGEMENTS}

This work was supported by the National Natural Science Foundation of China (No.51276105 and No.11561068), the Natural Science Foundation of Shaanxi Province (No. 2016JM4012), the Natural Science Foundation of Shaanxi Educational Committee (No.16JK1090), and the Shaanxi Science and Technology Research Development Programs of Key Innovative Industry Problem of China (No 2012ZKC10-1).

\section{DECLARATION OF CONFLICTING INTERESTS}

The authors declare that there is no conflict of interest.

\section{REFERENCES}

1. Abraao A.S., Lemos A.M., Vilela A., Sousa J.M., Nunes F.M., Influence of osmotic dehydration process parameters on the quality of candied pumpkins. Food Bioprod. Process., 2013, 91, 481-494.

2. Acosta O., Viquez F., Cubero E., Optimisation of low calorie mixed fruit jelly by response surface methodology. Food Qual. Prefer., 2008, 19, 79-85.

3. Azam M., Haq M.A., Hasnain A., Osmotic dehydration of mango cubes: Effect of novel gluten-based coating. Drying Technol., 2013, 31, 120-127.

4. Bejaoui M.A., Beltran G., Aguilera M.P., Jimenez A., Continuous conditioning of olive paste by high power ultrasounds: Response surface methodology to predict temperature and its effect on oil yield and virgin olive oil characteristics. LWT - Food Sci. Technol., 2016, 69, 175-184.

5. Chandra S., Kumari D., Recent development in osmotic dehydration of fruit and vegetables: A Review. Crit. Rev. Food Sci., 2015, 55, 552-561.

6. Corrêa J.L.G., Ernesto D.B., Alves J.G.L.F., Andrade R.S., Optimisation of vacuum pulse osmotic dehydration of blanched pumpkin. Int. J. Food Sci. Technol., 2014, 49, 2008-2014.

7. Dak M., Pareek N K., Effective moisture diffusivity of pomegranate arils undergoing microwave-vacuum drying. J. Food Eng., 2014, 122, 117-121.

8. Dong Q., Chen Z.D., Research progress on the osmotic dehydration of fruits and vegetables at home and abroad. Guangzhou Food Sci. Technol., 2004, 20, 129-132.

9. Erbay Z., Koca N., Kaymak-Ertekin F., Ucuncu M., Optimization of spray drying process in cheese powder production. Food Bioprod. Process., 2015, 93, 156-165.

10. Esan T.A., Sobukola O.P., Sanni L.O., Bakare H.A., Munoz L., Process optimization by response surface methodology and quality attributes of vacuum fried yellow fleshed sweetpotato (Ipomoea batatas L.) chips. Food Bioprod. Process., 2015, $95,27-37$.
11. Ghosh S., Das M.K., Optimization of the effect of gamma radiation on textural properties of different varieties of potato (Kufri Chandramukhi and Kufri Jyoti) and mango (Langra and Fazli) during storage by response surface methodology. Innov. Food Sci. Emerg. Technol., 2014, 26, 257-264.

12. Goula A.M., Lazarides H.N., Modeling of mass and heat transfer during combined processes of osmotic dehydration and freezing (Osmo-Dehydro-Freezing). Chem. Eng. Sci., 2012, 82, 52-61.

13. Huang S.Q., Fan L.P., A kinetics study on the combined drying of the osmotic dehydration and vacuum frying for the carrot chips. Modern Food Sci. Technol., 2013, 29, 223-225, 379.

14. Kaushik N., Srinivasa Rao P., Mishra H.N., Process optimization for thermal-assisted high pressure processing of mango (Mangifera indica L.) pulp using response surface methodology. LWT - Food Sci. Technol., 2016, 69, 372-381.

15. Lazo-Vélez M.A., Avilés-González J., Serna-Saldivar S.O., Temblador-Pérez M.C., Optimization of wheat sprouting for production of selenium enriched kernels using response surface methodology and desirability function. LWT - Food Sci. Technol., 2016, 65, 1080-1086.

16. Maran J.P., Sivakumar V., Thirugnanasambandham K., Sridhar R., Artificial neural network and response surface methodology modeling in mass transfer parameters predictions during osmotic dehydration of Carica papaya, L. Alex. Eng. J., 2013, 52, 507-516.

17. Nair G.R., Singh A., Kurian J., Raghavan V., Electro-osmotic dewatering of high moisture flax stems. Biosyst Eng., 2015, 133, $14-20$.

18. Nieto A.B., Vicente S., Hodara K., Castro M.A., Alzamora S.M., Osmotic dehydration of apple: Influence of sugar and water activity on tissue structure, rheological properties and water mobility. J. Food Eng., 2013, 119, 104-114 .

19. Nowacka M., Tylewicz U., Laghi L., Rosa M.D., Witrowa-Rajchert D., Effect of ultrasound treatment on the water state in kiwifruit during osmotic dehydration. Food Chem., 2014, 114, $18-25$.

20. Saxena A., Maity T., Raju P.S., Bawa A.S., Optimization of pretreatment and evaluation of quality of jackfruit (Artocarpus heterophyllus) bulb crisps developed using combination drying. Food Bioprod. Process., 2015, 95, 106-117.

21. Souraki B.A., Ghaffari A., Bayat Y., Mathematical modeling of moisture and solute diffusion in the cylindrical green bean during osmotic dehydration in salt solution. Food Bioprod. Process., 2012, 90, 64-71.

22. Wray D., Ramaswamy H.S., Development of a microwave-vacuum-based dehydration technique for fresh and microwave-osmotic (MWODS) pretreated whole cranberries (Vaccinium macrocarpon). Drying Technol., 2015, 33, 796-807.

23. Xin Y., Zhang M., Adhikari B., Effect of trehalose and ultrasound-assisted osmotic dehydration on the state of water and glass transition temperature of broccoli (Brassica oleracea L. var. botrytis L.). J. Food Eng., 2013, 119, 640-647.

24. Yadav B.S., Yadav R.B., Jatain M., Optimization of osmotic dehydration conditions of peach. J. Food Sci. Technol., 2012, 49, 547-555.

25. Yang H.J., Khan M.A., Han M.Y., Yu X.B., Bai X.J., Xu X.L., Zhou G.H., Optimization of textural properties of reduced-fat and reduced-salt emulsion-type sausages treated with high pres- 
sure using a response surface methodology. Innov. Food Sci. Emerg. Technol., 2016, 33, 162-169.

26. Zhang H., Feng Y.F., Zhang M., Sun J.C., Study of microwave and spouted bed even drying technology of lettuce. J. Food Sci. Biotechnol., 2012, 31, 402-410.
27. Zhao J.H., Hu R., Liu B., Ni Y.Y., Effect of osmotic dehydration pre-treatment on freezing rate and quality attributes of frozen mango. Trans of the CSAM, 2014, 45, 220-227.

Submitted: 7 September 2016. Revised: 19 December 2016. Accepted: 29 January 2017. Published on-line: 17 May 2017. 
